07

\title{
Синтез CVD-алмаза детекторного качества для радиационно-стойких детекторов ионизирующего излучения
}

\author{
(C) А.В. Красильников, ${ }^{1}$ Н.Б. Родионов, ${ }^{1}$ А.П. Большаков, ${ }^{2}$ В.Г. Ральченко, ${ }^{2}$ С.К. Вартапетов, ${ }^{3}$ \\ Ю.Е. Сизов, ${ }^{3}$ С.А. Мещанинов, ${ }^{1}$ А.Г. Трапезников, ${ }^{1}$ В.П. Родионова, ${ }^{1}$ В.Н. Амосов, ${ }^{1}$ \\ Р.А. Хмельницкий, ${ }^{1}$ А.Н. Кириченко ${ }^{1}$
}

\author{
1 Частное учреждение Государственной корпорации по атомной энергии „Росатом“ „Проектный центр ИТЭР“, \\ 123182 Москва, Россия \\ 2 Федеральный исследовательский центр Институт общей фризики им. А.М. Прохорова РАН, \\ 119991 Москва, Россия \\ ${ }^{3}$ ООО „Оптосистемы“, 108841 Троицк, Москва, Россия \\ e-mail: a.krasilnikov@iterrf.ru
}

Поступило в Редакцию 30 июля 2021 г.

В окончательной редакции 21 января 2022 г.

Принято к публикации 21 января 2022 г.

Сообщается о первом опыте создания в „Проектном центре ИТЭР“ на усовершенствованном СВЧ плазменном реакторе ARDIS-300 гомоэпитаксиальных структур посредством CVD-синтеза пленок монокристаллического алмаза. Методом осаждения из газовой фазы в смесях метан-водород в реакторе с СВЧ плазмой получены эпитаксиальные алмазные пленки высокого качества на легированных бором монокристаллических подложках алмаза. Структурное и примесное совершенство пленок охарактеризовано с применением спектроскопии комбинационного рассеяния света, фотолюминесценции и оптического поглощения. На пленках толщиной 70-80 $\mathrm{m}$, выращенных на подложках с проводимостью $p$-типа и концентрацией бора $\sim 100 \mathrm{ppm}$, служащих контактом, созданы прототипы радиационных детекторов, измерены их чувствительность и амплитудные спектры в потоках альфа-частиц и нейтронов с энергией 5.5 и 14.7 MeV соответственно. Показано, что при облучении альфа-частицами и нейтронами эффективность сбора заряда в синтезированном алмазе при приложенном поле $\sim 4 \mathrm{~V} / \mu \mathrm{m}$ достигает 94 и 91\% соответственно.

Ключевые слова: алмазные пленки, эпитаксия, алмазный детектор.

DOI: $10.21883 / J T F .2022 .04 .52247 .226-21$

\section{Введение}

Уникальные электрофизические свойства алмаза, как полупроводника c шириной запрещенной зоны $5.45 \mathrm{eV}[1]$ : высокие подвижности (до $\sim 4000 \mathrm{~cm}^{2} /(\mathrm{V} \cdot \mathrm{s})$ ) и скорость дрейфа $\left(\sim 10^{7} \mathrm{~cm} / \mathrm{s}\right)$ носителей заряда [2], высокое поле электрического пробоя, рекордно высокая теплопроводность (до $24(\mathrm{~W} / \mathrm{cm} \cdot \mathrm{K})$ ), широкий температурный диапазон работы (до $700^{\circ} \mathrm{C}$ ), высокая радиационная стойкость [3-5], высокая (в некоторых условиях до 100\%) эффективность сбора носителей заряда обеспечивают возможность создания радиационно-стойких детекторов ионизирующих излучений [6] и других высокозагруженных модулей алмазной электроники для применения в физике высоких энергий, в ряде отраслей, использующих ядерные технологии, в исследованиях термоядерной плазмы [7-14]. В конструкции детектора алмаз является твердотельной ионизационной камерой со сбором зарядов на электродах, располагаемых на двух сторонах алмазной пластины. Определяемый ядерными реакциями в алмазе амплитудный спектр алмазного детектора в потоке быстрых нейтронов, рожденных при синтезе дейтерия и трития [15], обеспечил эффективное применение алмазных детекторов для спектрометрии ДТ-нейтронов в термоядерных исследованиях на TFTR [7], JT-60U, JET [9] и на ряде других установок с ядерными технологиями.

Наивысшие характеристики детекторов достигаются при использовании монокристаллических пленок и пластин, выращиваемых в смесях метан-водород в СВЧ-плазменных в реакторах. Технология химического осаждения из газовой фазы (Chemical Vapor Deposition - CVD) обеспечивает низкое содержание примесей, прежде всего азота, и достаточно высокое кристаллическое совершенство [16-19].

Наиболее существенных успехов в синтезе алмазов с практически полным сбором зарядов (Charge Collection Efficiency - CCE) при облучении альфа-частицами с энергией $\sim 5.5 \mathrm{MeV}$ в последние 20 лет достигли в компании „Element Six“ (CCE $=95-100 \%$, энергетическое разрешение $\Delta E / E=0.4-1.2 \%$ при толщине пластин $\Delta=100-500 \mu \mathrm{m} \quad[20,21]), \quad$ Римском университете „Tor Vergata“ $(\mathrm{CCE} \sim 100 \%$ и $\Delta E / E=1.1 \%$, $\Delta=110 \mu \mathrm{m}[22])$, в компании „IIа Technology“, Сингапур (CCE $\sim 100 \%$ и $\Delta E / E=0.8 \%, \Delta=890 \mu \mathrm{m} \quad[23]$ ), в Университете Хоккайдо и AIST, Япония (CCE $\sim 100 \% \quad$ и $\Delta E / E=0.38 \%, \Delta=70-150 \mu \mathrm{m} \quad[24])$, ФГБНУ „ТИСНУМ““, Троицк, Россия $(\Delta E / E=0.56 \%$, $\Delta \sim 100 \mu \mathrm{m}[25,26])$. 
Типичная толщина алмазных пластин в детекторах составляет 200-500 $\mu \mathrm{m}$, что должно быть не менее длины собирания заряда (Charge Collection Distance, $\mathrm{CCD}$ ). Поскольку дрейфовая скорость носителей тока в алмазе начинает насыщаться в электрических полях порядка $1 \mathrm{~V} / \mu \mathrm{m}$, уменьшение толщины детектора до нескольких десятков микрометров позволяет значительно снизить необходимое напряжение смещения, что делает привлекательной конструкцию на тонкой пленке. Один из способов создания тонкопленочного алмазного детектора основан на эпитаксиальном осаждении методом CVD нелегированного алмаза на проводящую монокристальную алмазную подложку, легированную бором [7,27-29]. При этом подложка с проводимостью $p$-типа используется для эпитаксии нелегированного материала и как задний контакт детектора, а номинально нелегированный эпитаксиальный слой алмаза выступает в качестве активного слоя структуры, в котором концентрируется электрическое поле и движутся носители заряда, образованные ионизирующей частицей. Альтернативно задним контактом может служить заглубленный графитизированный слой, созданный ионной имплантацией [30].

В настоящей работе сообщается о первом в „Проектном центре ИТЭР“ опыте синтеза гомоэпитакисальных пленок CVD-алмаза толщиной несколько десятков микрометров на сильно легированных бором ( $p$-типа) НРНТ (high pressure high temperature) подложках. Исследованы эффективности сбора зарядов в синтезированных пленках и их детектирующая способность к альфа-частицам и нейтронам.

\section{CVD-синтез монокристаллических алмазных пленок}

Выращивание эпитаксиальных монокристаллических нелегированных алмазных пленок проводилось в специально модернизированном для синтеза алмаза детекторного качества СВЧ-плазменном реакторе ARDIS-300 (частота $2.45 \mathrm{GHz}$, мощность до $6 \mathrm{~kW}$ ) производства ООО „Оптосистемы“ [30].

Для реализации синтеза алмазного материала, обеспечивающего полный сбор зарядов, реактор был модернизован с целью снижения натекания в камеру из атмосферы и, как следствие, уменьшения концентрации фоновой примеси азота в газовой смеси.

При синтезе алмазных пленок комплекс модернизированного CVD-реактора ARDIS-300 позволяет напрямую управлять следующими параметрами: независимым напуском водорода, метана, азота, кислорода и борсодержащего газа или паров борсодержащей жидкости в водороде (в перспективе) в камеру установки, давлением газовой смеси в ней, мощностью СВЧ излучения и положением подложки по высоте. От этих параметров зависят: содержание указанных выше газов в газовой смеси, температура подложки и соответственно скорость роста алмазной пленки. Давление в камере поддерживается спиральным безмасляным насосом, а высокий вакуум достигается с помощью турбомолекулярного насоса. Предельно достижимый вакуум в реакторе составляет $5 \cdot 10^{-7}$ Torr. Контроль давления в камере осуществляется датчиками двух типов: Пирани и емкостным.

В качестве реагентов использовали высокочистые газы: водород (чистота 99.99999\%) и метан (99.9999\%). Температура подложки в процессе синтеза измерялась двухцветным ИК пирометром Sensortherm Metis M3.

Уровень натекания из атмосферы в реактор не превышал $2.5 \cdot 10^{-6}$ Torr $\cdot 1 / \mathrm{s}$, что в CVD-процессе обеспечивает в газовой смеси в камере объемом $5 l$ концентрацию молекул азота $\mathrm{N}_{2}$ около 2 ppm. При типичной концентрации метана (4\%) отношение N/C составляло $\sim 100$ ppm. C учетом того что коэффициент встраивания атомов азота $(\mathrm{N} / \mathrm{C})_{\text {dia }} /(\mathrm{N} / \mathrm{C})_{\text {gas }}$ в растущий алмаз в CVD-процессе составляет $\sim 2 \cdot 10^{-4}[31,32]$, следует ожидать концентрацию азота в эпитаксиальном материале на уровне $20 \mathrm{ppb}$ (вклад в концентрацию азота в алмазе от примеси в рабочем газе на 2 порядка меньше, $\sim 0.1 \mathrm{ppb}$ ).

В качестве подложек для эпитаксии использовались проводящие легированные бором пластины алмаза (В21, В22, В23), синтезированные в аппаратах высокого давления и высоких температур (метод High Pressure-High Temperature - НРНТ), с концентрацией бора $\approx 100$ ppm (для алмаза $1 \mathrm{ppm}=1.76 \cdot 10^{17} \mathrm{~cm}^{-3}$ ). Для определения концентрации одиночных замещающих атомов азота в нейтральном зарядовом состоянии $\left(N_{s}^{0}\right)$, исторически называемых С-дефектами, в выращенном эпитаксиальном материале одна пленка была выращена на нелегированной НРНТ-подложке (Н03) с низким содержанием азота (тип IІа). Синтез пленки Н03 проводился в условиях, аналогичных условиям для получения образцов B21-B23.

Полированные подложки имели размеры $4.5 \times 4.5 \times 0.5 \mathrm{~mm}$ и ориентацию ростовых граней (100). Шероховатость поверхностей составляла не более $5 \mathrm{~nm}$ на основании измерений с использованием атомно-силового микроскопа Solver Next (NT-MDT). Для отобранных подложек максимальное отклонение ориентации рабочей грани от кристаллографической плоскости (100) не превышало 3 градусов согласно измерениям на рентгеновском дифрактометре „Bruker D8 Discover A25 DaVinsiDesign“. Перед загрузкой в камеру реактора подложки отжигали в муфельной печи на воздухе при температуре $590^{\circ} \mathrm{C}$ для удаления неалмазной фазы углерода, кипятили в растворе бихромата калия $\left(\mathrm{K}_{2} \mathrm{Cr}_{2} \mathrm{O}_{7}\right)$ в концентрированной серной кислоте $\left(\mathrm{H}_{2} \mathrm{SO}_{4}\right)$ для удаления возможных загрязнений на поверхности после полировки и обрабатывали в изопропиловом спирте в ультразвуковой ванне. Процесс роста проводили при условиях, описанных в табл. 1. В результате получены алмазные слои толщиной от 50 до $80 \mu \mathrm{m}$. Скорость роста пленки В23 была выше в связи с тем, что подложка располагалась на большей высоте, ближе по отношению к плазме. 
Таблица 1. Условия синтеза пленок: давление $p$, концентрация метана в газе $\left[\mathrm{CH}_{4}\right]$, температура подложки $T_{\mathrm{s}}$, СВЧ мощность $P_{\mathrm{MW}}$, время осаждения $t_{\mathrm{g}}$, скорость роста $G R$, толщина пленки $d$

\begin{tabular}{c|c|c|r|r|r|r|c}
\hline $\begin{array}{c}\text { № } \\
\text { образца }\end{array}$ & $\begin{array}{c}p, \\
\text { Torr }\end{array}$ & $\begin{array}{c}{\left[\mathrm{CH}_{4}\right]} \\
\%\end{array}$ & $\begin{array}{c}T_{\mathrm{s}}, \\
{ }^{\circ} \mathrm{C}\end{array}$ & $\begin{array}{c}P_{\mathrm{MW}}, \\
\mathrm{W}\end{array}$ & $\begin{array}{c}t_{\mathrm{g}}, \\
\mathrm{h}\end{array}$ & $\begin{array}{c}G R, \\
\mu \mathrm{m} / \mathrm{h}\end{array}$ & $\begin{array}{c}d, \\
\mu \mathrm{m}\end{array}$ \\
\hline $\mathrm{B} 21$ & 170 & 4 & 940 & 5500 & 23.5 & 3.0 & 70 \\
$\mathrm{~B} 22$ & 170 & 4 & 940 & 5500 & 23.5 & 3.2 & 75 \\
$\mathrm{~B} 23$ & 170 & 4 & 965 & 3600 & 20.0 & 4.0 & 80 \\
$\mathrm{H} 03$ & 140 & 1.5 & 1050 & 3500 & 66 & $<1.0$ & 51
\end{tabular}

Поверхности синтезированных образцов исследовались методом дифференциальной интерференционноконтрастной микроскопии (ДИК) по Номарскому на оптическом микроскопе Olympus BX43. Пленка B21 имела относительно гладкую поверхность слоевого роста, макродефекты типа пирамид роста - хиллоков (hillocks), часто встречающиеся при росте CVD-алмаза [33], отсутствовали. Поверхность пленки В22 более развитая, на ней обнаруживалось некоторое количество хиллоков и поликристаллическая пленка на боковых гранях, а на образце В23 - много пирамид роста по всей поверхности. Морфология пленки В21 оценена, как наилучшая в серии.

Изготовленные алмазные пленки подвергали кипячению в растворе бихромата калия в концентрированной серной кислоте в течение $1 \mathrm{~h}$ для удаления аморфного углерода, который мог осесть на образцах в процессе остывания образца после выключения плазмы, промывали дистиллированной водой, затем кипятили в деионизованной воде и обрабатывали изопропиловым спиртом в ультразвуковой ванне. Один из образцов (В23) был отшлифован и отполирован до толщины эпитаксиального слоя $60 \mu \mathrm{m}$ (удалено $20 \mu \mathrm{m}$ ).

\section{2. Оптическая спектроскопия}

Фазовую чистоту пленок и наличие оптически активных дефектов в образцах исследовали с помощью спектроскопии комбинационного рассеяния (КР) и фотолюминесценции (ФЛ) на спектрометре „Renishaw in Via Reflex“ при возбуждении лазером с длиной волны $532 \mathrm{~nm}$ в геометрии обратного рассеяния. Спектр КР для образца В21 показан на рис. 1, $a$. В спектре присутствует единственная характерная для алмаза линия с максимумом на частоте $1332.5 \mathrm{~cm}^{-1}$. Ее полная ширина на половине высоты (ПШПВ) характеризует в основном структурное совершенство материала, наличие упругих напряжений из-за дислокаций и других протяженных дефектов. Величина ПШПВ составила 2.2, $2.4,2.7$ и $2.3 \mathrm{~cm}^{-1}$ для пленок В21, В22, В23 и $\mathrm{H} 03$ соответственно.

После измерений в качестве детекторов (см. ниже разд. 3) с эпитаксиальных пленок В21 и В22 химическим способом были удалены контакты, у пленки В21 был отполирован торец, а у пленки В22 - ростовая поверхность. Их спектры ФЛ до и после полировки (у пленки В21 на расстоянии $5 \mu \mathrm{m}$ от ростовой поверхности) показаны на рис. $1, b$. В спектрах кроме компонент КР 1-го и 2-го порядка сосуществуют два оптических центра, образованных $\mathrm{NV}$-дефектами (одиночный замещающий атом азота рядом с вакансией углерода): $\mathrm{NV}^{0}$ (нейтральный) и $\mathrm{NV}^{-}$(отрицательно заряженный) c бесфононными линиями 575 и $638 \mathrm{~nm}$ соответственно и их фононными повторениями. Концепция уровня Ферми не полностью применима к широкозонным полупроводникам с низким уровнем легирования, поэтому в алмазе одинаковые дефекты обнаруживаются в нескольких зарядовых состояниях [34]. Зарядовое состояние $\mathrm{NV}$-дефекта определяется, главным образом, локальным окружением [35]. Находящийся недалеко от него атом $N_{s}^{0}$ способен передать ему свой пятый электрон (при этом он сам становится положительно заряженным $-N_{s}^{+}$), образуя оптический центр $\mathrm{NV}^{-}$, доминирующий в ФЛ на рис. $1, b)$. Хотя, как показано в работе [36], в ФЛ исследовании при возбуждении в диапазоне длин волн $450-610 \mathrm{~nm}$ популяция $\mathrm{NV}^{-}$центров не может быть больше $75 \%$. Тем не менее спектры на рис. 1 указывают на наличие в эпитаксиальном материале атомов $N_{s}^{0}$, которые сами не проявляются в люминесценции, но влияют на зарядовое состояние люминесцирующих $\mathrm{NV}$-дефектов.

Из рис. $1, b$ видно, что интенсивности ФЛ пленок В21 и В22 (по отношению к пику КР 1-го порядка) отличаются в несколько раз. Судя по всему, это связано с разной морфологией роста и соответственно различием вероятности встраивания радикалов азота в процессе роста пленки. Интенсивность ФЛ пленок В21, В22 и В23 коррелирует с величиной ПШПВ: она максимальна для пленки В23 (значение ПШПВ для нее составляет $2.7 \mathrm{~cm}^{-1}$ ) и минимальна для пленки В21 (значение ПШПВ для нее составляет $2.2 \mathrm{~cm}^{-1}$ ). Примечательно, что после полировки пленок В21 и В22 интенсивность ФЛ в них заметно снижается (рис. $1, b$ ). Это входит в противоречие с наблюдениями в работе [37], в которой обнаружено усиление люминесценции дефектов после полировки алмаза. Возможно, это зависит от режима полировки. При аккуратной полировке в мягком направлении в приповерхностном слое происходит только пластическое течение материала, наоборот, при обработке с хрупким удалением материала генерируются дефекты, в том числе такие точечные дефекты, как вакансии, способные в реакции с $\mathrm{N}_{s}$ образовывать $\mathrm{NV}$-дефекты [38].

Одиночный замещающий азот обнаруживается в оптическом поглощении. В кристаллах типа $\mathrm{Ib}$ поглощение на электронных переходах начинается с энергии квантов $1.7 \mathrm{eV}$ и плавно нарастает вплоть до фундаментального края. При низких концентрациях $\mathrm{N}_{s}$ на этом фоне проявляется полоса поглощения с максимумом вблизи $270 \mathrm{~nm}$ и ПШПВ около $20 \mathrm{~nm}$, интенсивность которой пропорциональна концентрации $N_{s}^{0}$ [39]. Впрочем, было 

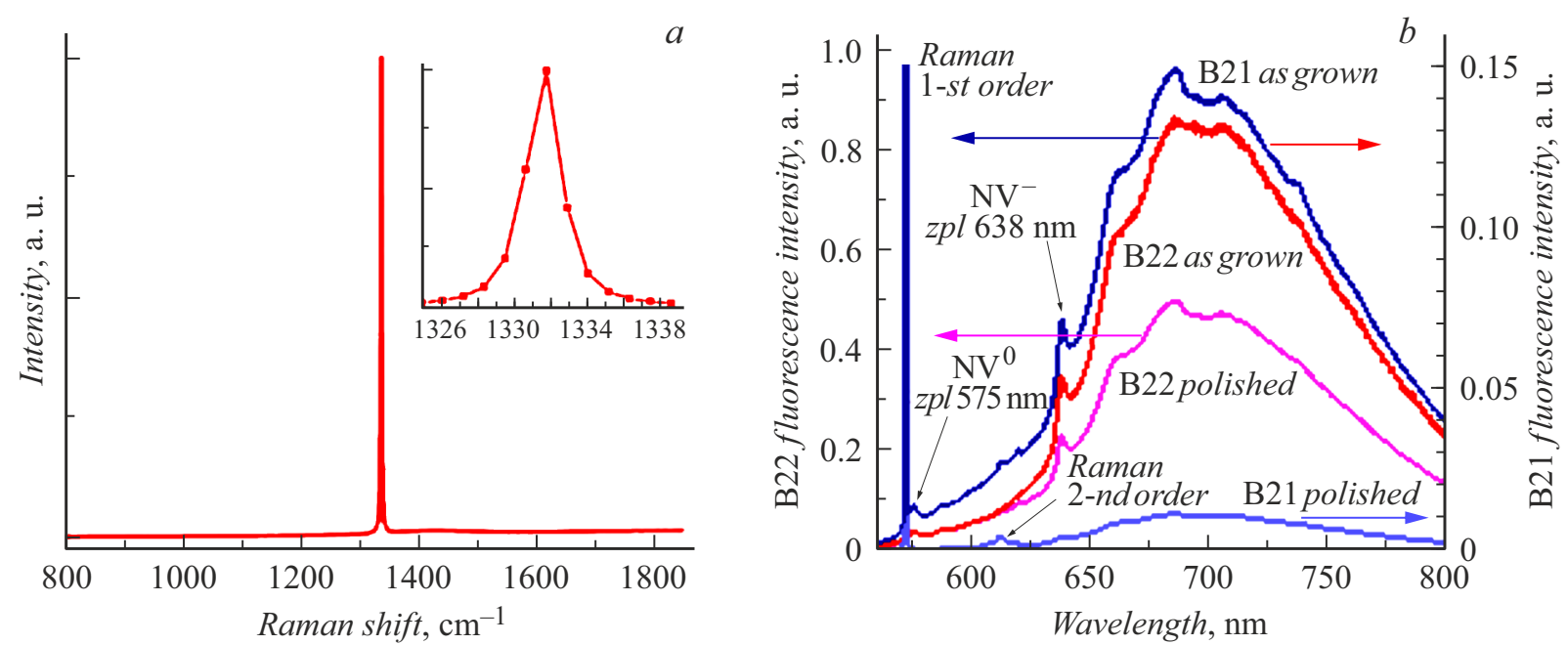

Рис. 1. $a$ - спектр КР образца В21. На вставке - спектр КР в диапазоне $1320-1340 \mathrm{~cm}^{-1} ; b-$ спектры ФЛ эпитаксиальных пленок В21 (правая шкала) и В22 (левая шкала) до и после полировки. Спектры нормированы к единице по амплитуде пика КР 1-го порядка на длине волны $573 \mathrm{~nm}$. Возбуждение лазером с длиной волны $532 \mathrm{~nm}$.

высказано предположение, что в материале с азотом в виде доминирующих $N_{s}^{0}$ эта полоса связана с образованием отрицательно заряженного $N_{s}^{-}$по реакции $N_{s}^{0}+h v \rightarrow N_{s}^{-}+h^{+}$, где $h^{+}$- дырка в валентной зоне [40]. В любом случае оптическое поглощение в этой полосе принято использовать для определения концентрации $N_{s}^{0}$ в алмазе.

На прозрачном образце Н03 было измерено оптическое поглощение до и после эпитаксии в диапазоне длин волн 230-350 nm с помощью спектрофотометpa LAMBDA $850+$ (PerkinElmer, USA) и с диафрагмой диаметром $4 \mathrm{~mm}$. В наших измерениях максимум пика на самом деле приходится на 272-275 nm. На основании калибровки из работы [40] средняя концентрация $N_{s}^{0}$ в подложке Н03 составляет $95 \pm 8 \mathrm{ppb}$ $\left(1 \mathrm{ppb}=1.76 \cdot 10^{14} \mathrm{~cm}^{-3}\right)$. После эпитаксии не было обнаружено увеличения пика с максимумом вблизи $270 \mathrm{~nm}$. С учетом чувствительности измерений и других ошибок можно утверждать, что концентрация $N_{s}^{0}$ в эпитаксиальной пленке Н03 толщиной $51 \pm 11 \mu \mathrm{m}$ и синтезированных при близких условиях пленках В21-В23, по крайней мере, не превышает 50 ppb.

\section{3. Эффективность сбора заряда в пленках}

Для измерения эффективности сбора зарядов (электронов и дырок), рождаемых в алмазе частицами высоких энергий, на ростовую сторону пленки и свободную сторону проводящей подложки магнетронным напылением при температуре образца $250^{\circ} \mathrm{C}$ были нанесены сплошные металлические контакты из платины толщиной $35 \mathrm{~nm}$. Чувствительный элемент с нанесенными контактами монтировался в специальный корпус.
Измерение амплитудных спектров детектора было проведено при облучении альфа-частицами от образцового источника ${ }^{241} \mathrm{Am}$ с энергией $\sim 5.5 \mathrm{MeV}$ и активностью $\sim 30 \mathrm{kBq}$ при атмосферном давлении и расположении детектора на расстоянии $\sim 2 \mathrm{~mm}$ от источника и нейтронами на генераторе ИНГ-07Т. Потери внутри образцового источника альфа-частиц малы, так как он не покрыт защитным слоем, а активный слой очень тонкий. Расхождение с теоретическим значением энергии альфа-частицы ${ }^{241} \mathrm{Am}$ по нашим оценкам не более $50 \mathrm{keV}$. Потери на контактах детекторов незначительные (не более $2 \mathrm{keV}$ ), при этом уширение пика энергетического спектра на контактах менее $0.1 \%$. $2 \mathrm{~mm}$ воздуха между альфа-источником и детектором вызывает потери энергии альфа-частиц $\sim 200 \mathrm{keV}$, при этом пик уширяется в 3.9 раз. Это измерено на детекторе (Eg13), относительно которого приведены результаты, но в составе другого измерительного тракта. Ускоряющее напряжение ИНГ-07Т составляло $130 \mathrm{kV}$ при токе $100 \mathrm{~mA}$. Детекторы располагались на расстоянии $5 \mathrm{~cm}$ от мишени нейтронного генератора вдоль линии ускоренного дейтерий-тритиевого пучка генератора в потоке нейтронов с энергией $\sim 14.7 \mathrm{MeV}$ и плотностью $\sim 1.1 \cdot 10^{6} \mathrm{~cm}^{-2} s^{-1}$. Состав измерительного тракта: зарядочувствительный предусилитель Canberra 2004, усилитель-формирователь Ortec 673, блок питания Ortec 556, спектрометрический АЦП Ortec 926.

Измеренные амплитудные спектры сравнивались со спектрами образцового алмазного детектора Eg13 (изготовитель „Проектный центр ИТЭР“ из CVD-алмаза компании „Element $\mathrm{Six}^{\text {“ }}$ ), у которого эффективность сбора заряда составляла $\sim 98 \%$, а энергетическое разрешение $\Delta E / E \sim 0.8 \%$. Амплитудные спектры детекторов в потоке альфа-частиц источника ${ }^{241} \mathrm{Am}$ на пленках В21, В22 представлены на рис. 2, $a$ на пленке В23 - на 

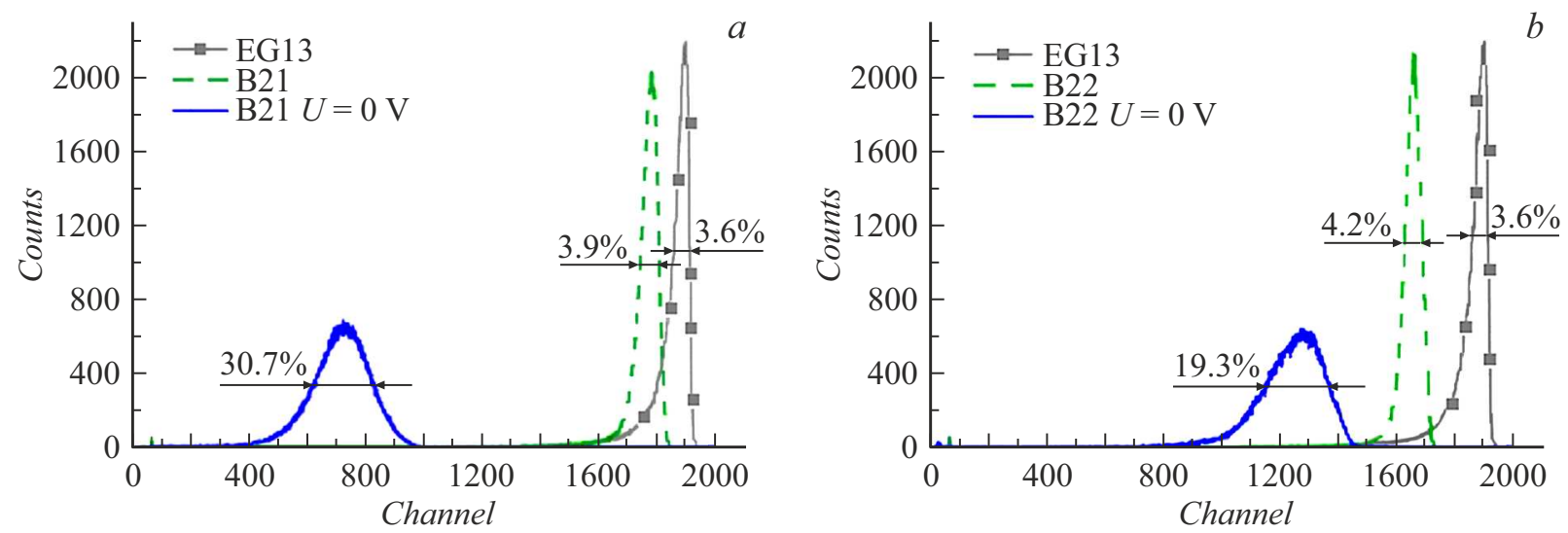

Рис. 2. Амплитудные спектры детекторов на образцах В21 (a) и В22 (b) под альфа-излучением ${ }^{241}$ Аm в сравнении с образцовым $\operatorname{Eg} 13$ (серый цвет) при напряжении $U=-60 \mathrm{~V}$ (со стороны подложки) на детекторах (штриховая линия) и без напряжения питания $U=0 \mathrm{~V}$ (синий цвет в онлайн версии).

$a$

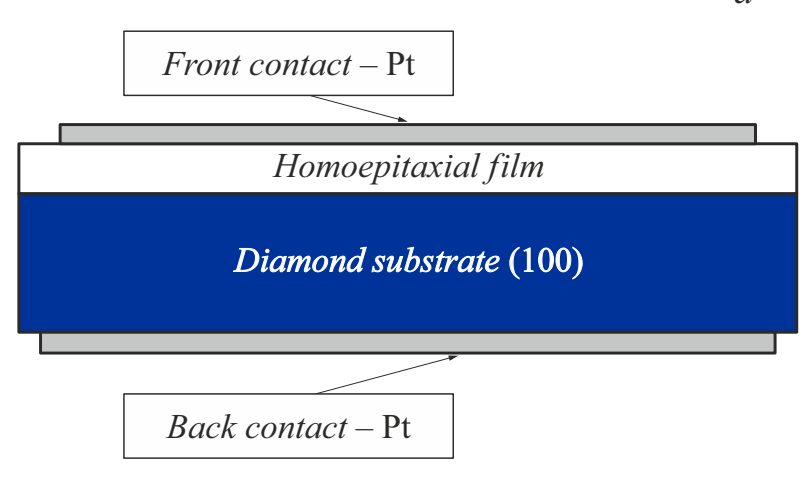

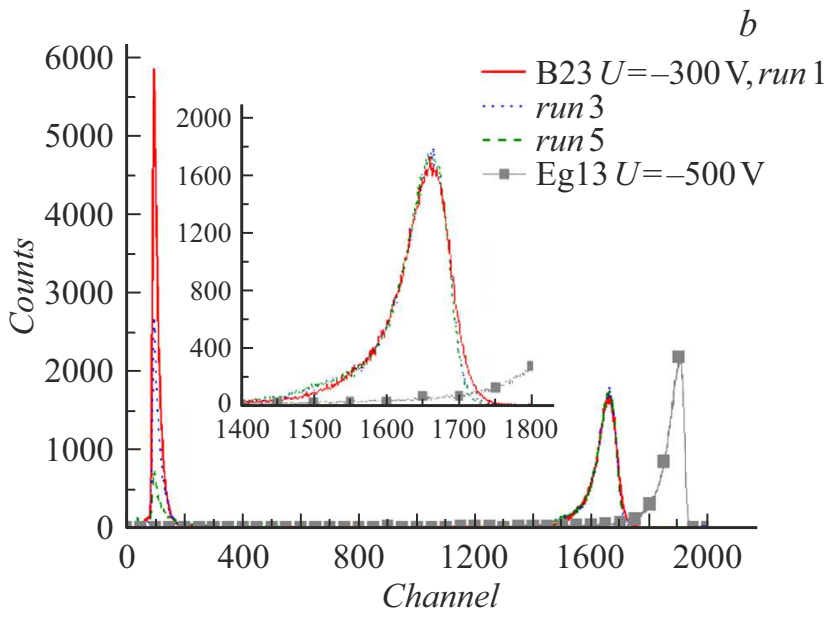

Рис. 3. Схематическое изображение структуры детектора $(a) ; b-$ амплитудные спектры детектора В23 под альфа-излучением ${ }^{241} \mathrm{Am}$ в трех из пяти последовательных измерений при напряжении смещения $U=-300 \mathrm{~V}$ (показаны разными цветами, справа). Спектр для образцового Eg13 (серый цвет) получен при смещении $U=-500 \mathrm{~V}$. На вставке: спектры от детектора В23 в узком диапазоне энергий. Время набора одного спектра $t=300 \mathrm{~s}$.

рис. 3. Измеренные полные ширины на половине высоты амплитудных пиков пленок В21, В22 и В23 составили $3.9,4.2$ и $4.24 \%$ соответственно, что близко к величине ПШПВ $3.6 \%$ для образцового кристалла $\operatorname{Eg} 13$, и определялись энергетическим спектром альфа-частиц после прохода через $\sim 2 \mathrm{~mm}$ воздуха и энергетическим разрешением соответствующей пленки. ПШПВ энергетического спектра альфа-источника на детекторах в наших измерениях может быть оценена из измерений Eg13 как $\left((3.6 \%)^{2}-(0.8 \%)^{2}\right)^{0.5}=3.4 \%$. Соответственно энергетические разрешения пленок В21-В23 могут быть оценены квадратичным вычитанием из ширины их амплитудных спектров ширины энергетического распределения альфа-частиц (3.4\%) величинами порядка 1.7 , 2.45 и $2.5 \%$ соответственно. Отметим, что длина пробега альфа-частиц в алмазе составляет около $12 \mu \mathrm{m}$, т.е. генерация электрон-дырочных пар происходила только в прианодной части объема детектора.
Основное отличие характеристик (например, эффективный сбор заряда ССЕ) образцового алмазного детектора Eg13 (изготовлен на пластине CVD-алмаза компании „Element Six“) от образцов B21-B23, рассмотренных в настоящей работе, связано с разным содержанием азота в CVD-алмазе. „Element Six“ декларирует содержание азота в алмазе электронного качества порядка $1 \mathrm{ppb}$, тогда как в полученных образцах удалось экспериментально показать, что содержание азота составляет не более $50 \mathrm{ppb}$, что позволяет отнести материал пленки к материалу детекторного качества. Следует отметить, что выбранный в качестве образцового кристалл Eg13, хотя и характеризуется низким содержанием примесного азота и высоким кристаллическим совершенством, не обладает рекордными характеристиками в качестве материала электронного качества. Поэтому результаты, полученные при его использовании, отличаются от приведенных в литературе для наилучших образцов. 
Максимум сигнала для образцового Eg13 приходится на канал \#1899. По смещению пика в амплитудном спектре при генерации электронно-дырочных пар в прианодном пространстве пленок при напряжении смещения $60 \mathrm{~V}$ эффективности сбора заряда в пленках В21, В22 составили 92, 86\% соответственно, при погрешности измерений $\pm 0.2 \%$. В пленке В23 эффективность сбора заряда составила $86 \%$ при напряжении смещения $300 \mathrm{~V}$. Следует отметить стабильность формы амплитудного спектра: так, в серии из пяти последовательных интервалов длительностью $300 \mathrm{~s}$ каждый для В23 спектры были практически идентичными, что указывает на относительно низкий уровень концентрации центров захвата заряда в пленках (на рис. 3 приведены спектры трех из пяти последовательных измерений). Для определения величины ПШПВ в спектрах КР использовалось программное обеспечение WiRE 5.1, поставляемое вместе со спектрометром. Форма линии аппроксимировалась распределением Фойгта. На профиле линии КР умещалось 6 точек. Среднеквадратичное отклонение рассчитанных ПШПВ составило $0.1 \mathrm{~cm}^{-1}$. Следует отметить, что значения ПШПВ получаемых спектров несколько завышены. Так, ПШПВ линии неона калибровочной неоновой лампы, поставляемой вместе с прибором, составило $1.3 \mathrm{~cm}^{-1}$. Но, несмотря на это, представленные в табл. 2 и на рис. 1 характеристики синтезированных алмазных пленок позволяют сделать вывод о корреляции роста эффективности сбора заряда с уменьшением ширины пика КР.

Легированные бором подложки, хотя и не отличались кардинально по концентрации бора, которая была порядка $100 \mathrm{ppm}$, тем не менее заметно отличались по ширине пика КР $\Delta v s$. Таким образом, подложки различались степенью дефектности. Согласно данным, представленным в табл. 2, ширина пика КР пленки $\Delta v_{f}$ возрастает с величиной $\Delta v_{s}$, т.е. пленка наследует в какой-то степени дефектность подложки. Лучший образец В21 как раз соответствует подложке с минимальной шириной пика КР. Близость величин ширины пика КР для этого образца В21 и для образца Eg13 от Element Six еще раз указывает на наиболее высокое качество первого по отношению к образцам В22 и В23.

Следует отметить, что и интенсивность ФЛ пленок В21 и В22 (по отношению к пику КР 1-го порядка) в диапазоне $600-800 \mathrm{~nm}$ (рис. 1), связанная с наличием

Таблица 2. Эффективность сбора заряда $\varepsilon$, ширины пика КР алмазной пленки $\Delta v_{f}$ и подложки $\Delta v_{s}$

\begin{tabular}{c|c|c|c}
\hline $\begin{array}{c}\text { Номер } \\
\text { образца }\end{array}$ & $\begin{array}{c}\text { Эффективность } \\
\text { сбора заряда } \varepsilon, \%\end{array}$ & $\begin{array}{c}\text { Ширина пика КР } \\
\text { алмазной пленки } \\
\Delta v_{f}, \mathrm{~cm}^{-1}\end{array}$ & $\begin{array}{c}\text { Ширина пика КР } \\
\text { подложки } \\
\Delta v_{s}, \mathrm{~cm}^{-1}\end{array}$ \\
\hline Eg13 & 98 & 2.2 & $\mathrm{n} / \mathrm{a}$ \\
В21 & 92 & 2.2 & 3.8 \\
В22 & 86 & 2.4 & 4.0 \\
В23 & 86 & 2.7 & 5.7
\end{tabular}

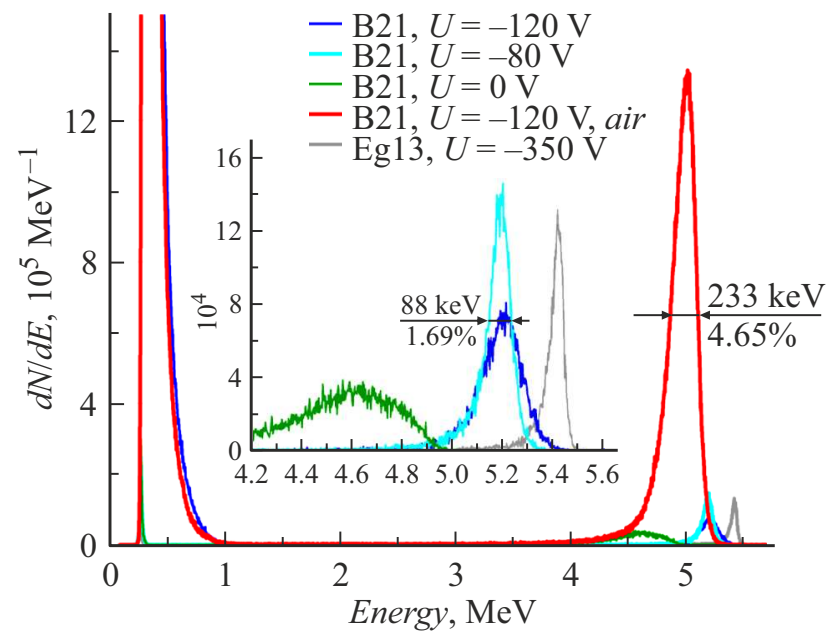

Рис. 4. Амплитудные спектры заново изготовленного детектора с пленкой В21 в потоке альфа-частиц ${ }^{241} \mathrm{Am}$ в вакууме (синий, голубой, зеленый (в онлайн версии)) и на воздухе (красный (в онлайн версии)) в сравнении с Eg13 в вакууме (серый (в онлайн версии)).

азот- вакансионных комплексов, также коррелирует с эффективностью сбора заряда: наилучшая эффективность наблюдается у образца В21, в котором интенсивность фотолюминесценции минимальна.

Эффективность сбора заряда зависит в значительной мере от примесей и точечных дефектов, выступающих в качестве ловушек и центров рассеяния носителей заряда.

С целью оценки влияния контактов на ССЕ и энергетическое разрешение детектора платиновые контакты образца В21 были удалены химическим способом и нанесены заново. После этого образец с пленкой В21 с заново изготовленными платиновыми контактами толщиной $35 \mathrm{~nm}$ был вновь помещен в детекторный корпус и были зарегистрированы его амплитудные спектры в потоках альфа-частиц того же источника ${ }^{241} \mathrm{Am}$ в аналогичных предыдущим измерениям условиях на воздухе и в вакууме (при расстояниях между источником и детектором 2 и $20 \mathrm{~mm}$ соответственно). Зарегистрированные амплитудные спектры В21 с новыми контактами (представлены на рис. 4) продемонстрировали ССЕ $\sim 94 \%$ и энергетическое разрешение $88 \mathrm{keV}$ (1.69\%).

Для оценки снизу эффективности сбора зарядов при равномерной генерации электрон-дырочных пар во всем объеме синтезированных алмазных пленок были зарегистрированы амплитудные спектры детекторов с пленками В21, В22 и В23 в потоках нейтронов с энергией $14.7 \mathrm{MeV}$ и полной шириной на половине высоты энергетического распределения $\sim 320 \mathrm{keV}$. Полученные амплитудные спектры, определяемые реакциями $\mathrm{C}(n, n) \mathrm{C}, \mathrm{C}(n, \alpha) 2 \alpha$ и $\mathrm{C}(n, \alpha) \mathrm{Be}[13]$ (рис. 5), продемонстрировали высокую эффективность сбора заряда в синтезированных пленках В21, В22 и В23 и при равномерно распределенной по объему генерации электрон-дырочных пар. Измеренные сдвиги положения 

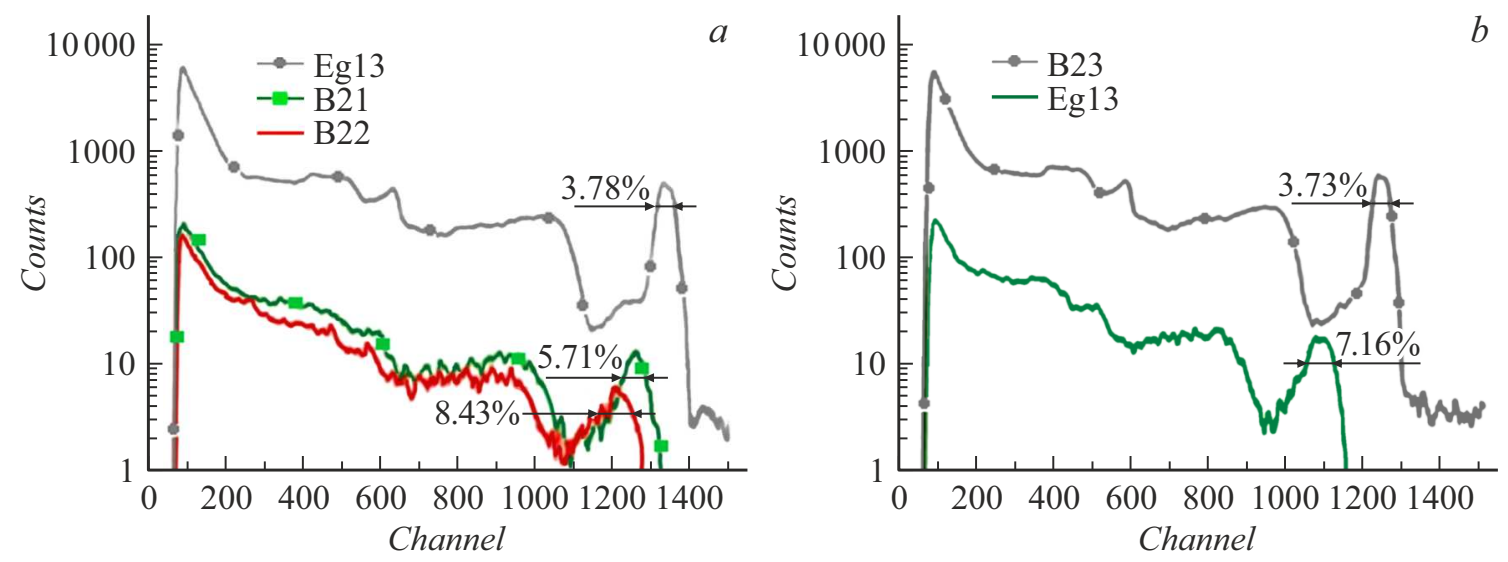

Рис. 5. Амплитудные спектры детекторов с пленками В21 и В22 (a) и В23 (b) в потоке нейтронов с энергией $14.7 \mathrm{MeV}$ нейтронного генератора ИНГ-07Т на расстоянии $5 \mathrm{~cm}$ от его мишени.

пика амплитудного спектра, обусловленного реакцией $\mathrm{C}(n, \alpha) \mathrm{Be}$ (правые пики в спектрах), позволили оценить снизу эффективности сбора зарядов в В21, В22 и В23 величинами 91, 85 и 85\% соответственно. Существенное увеличение ширин $\mathrm{C}(n, \alpha) \mathrm{Be}$ пиков (рис. 5) как по сравнению с образцовым Eg13, так и по сравнению со спектрами в потоках альфа-частиц(рис. 2-4) может быть связано как с существенно более низкой статистикой, так и с тем, что в тонких пленках заметная часть заряженных продуктов реакции $\mathrm{C}(n, \alpha) \mathrm{Be}$, генерируемых вблизи границы пленки с подложкой, может вылетать из пленки, не отдав всю энергию.

\section{Заключение}

В модернизированном СВЧ-плазмохимическом реакторе на легированных бором монокристаллических подложках алмаза в смесях $\mathrm{CH}_{4} / \mathrm{H}_{2}$ синтезированы гомоэпитаксиальные нелегированные алмазные пленки детекторного качества толщиной до $80 \mu \mathrm{m}$. Показано, что эффективность сбора заряда растет с уменьшением ширины алмазной линии в спектре комбинационного рассеяния света (улучшается кристаллическое совершенство) в пленке и с уменьшением интенсивности ФЛ. Экспериментально продемонстрировано, что эффективности сбора зарядов, порождаемых в синтезированном алмазе потоками альфа-частиц с энергией $5.5 \mathrm{MeV}$ и нейтронов с энергией $14.7 \mathrm{MeV}$ достигает 94 и 91\% соответственно. Созданные на основе пленок образцы радиационных детекторов продемонстрировали высокую стабильность характеристик при регистрации альфа-частиц и нейтронов.

Несмотря на наличие ФЛ, связанной с азот-вакансионными комплексами, синтезированные пленки продемонстрировали высокую эффективность сбора заряда, что позволяет использовать их для изготовления детекторов.

\section{Финансирование работы}

Работа выполнена по государственному контракту № H.4ф.241.09.20.1087 от 05.06.2020.

\section{Конфликт интересов}

Авторы заявляют, что у них нет конфликта интересов.

\section{Список литературы}

[1] A. Alekseyev, G. Martin. Nucl. Instrum. Methods Phys. Res., A 417, 400 (1998). DOI: 10.1016/S0168-9002(98)00762-1

[2] H. Frais-Kolbl, E. Griesmayer, H. Kagan, H. Pernegger. IEEE Transactions Nucl. Sci., NS-51, 3833 (2004). DOI: $10.1109 /$ TNS.2004.839366

[3] H. Kagan. Nucl. Instrum. Methods Phys. Res., A 541, 221 (2005). DOI: 10.1016/j.nima.2005.01.060

[4] M. Bruzzi, S. Miglio, S. Pirollo, S. Sciortino. Diamond and Related Mater., 10 (3-7), 601 (2001).

[5] C. Bauer, I. Baumann, C. Colledani, J. Conway, P. Delpierre, F. Djama, M. Zoeller. Nucl. Instrum. Methods Phys. Res. Section A: Accelerators, Spectrometers, Detectors and Associated Equipment, 383 (1), 64 (1996).

[6] E.A. Konorova, S.F. Kozlov. Sov. Phys. Semicond, 4, 1600 (1971).

[7] A.V. Krasilnikov, E.A. Azizov, A.L. Roquemore, V.S. Khrunov, K.M. Young. Rev. Sci. Instrum., $68(1)$, 553 (1997).

[8] A.V. Krasilnikov, V.N. Amosov, P. van Belle, O.N. Jarvis, G.J. Sadler, Nucl. Instrum. Meth. Phys. Res., A 476 (1), 516 (2002).

[9] M. Marinelli, E. Milani, G. Prestopino, A. Tucciarone, C. Verona, G. Verona-Rinati, M. Angelone, D. Lattanzi, M. Pillon, R. Rosa, E. Santoro. Appl. Phys. Lett., 90, 183509 (2007).

[10] R.S. Balmer, J.R. Brandon, S.L. Clewes, H.K. Dhillon, J.M. Dodson, I. Friel, P.N. Inglis, T.D. Madgwick, M.L. Markham, T.P. Mollart, N. Perkins, G.A. Scarsbrook, D.J. Twitchen, A.J. Whitehead, J.J. Wilman, S.M. Woollard. J. Phys.: Condens. Matter, 21, 364221 (2009). 
[11] M. Pillon, M. Angelone, A. Krasa, A.J.M. Plompen, P. Schillebeeckx, M.L. Sergi. Nucl. Instrum. Meth. Phys. Res., A 640, 185 (2011).

[12] P. Barberet, M. Pomorski, G. Muggiolu, E. Torfeh, G. Claverie, C. Huss, S. Saada, G. Deves, M. Simon, H. Seznec. Appl. Phys. Lett., 111, 243701 (2017).

[13] H. Kagan, A. Alexopoulos, M. Artusot, F. Bachmair et al. Nucl. Instrum. Meth. Phys. Res., A 924, 297 (2019).

[14] C.Y. Lee, C.M. Ban, H.R. Lee, K.N. Choo, B.H. Jun. Appl. Rad. Isotopes, 152, 25 (2019).

[15] A.V. Krasilnikov. Issues Atom. Sci. Tech., 36 (1), (1995).

[16] A. Tallaire, J. Achard, F. Silva, O. Brinza, A. Gicquel. Comptes Rend. Phys., 14, 169 (2013). https://doi.org/10.1016/j.crhy.2012.10.008

[17] S. Nad, Y. Gu, J. Asmussen. Diam. Relat. Mater., 60, 26 (2015).

[18] A.P. Bolshakov, V.G. Ralchenko, V.Y. Yurov, A.F. Popovich, I.A. Antonova, A.A. Khomich, E.E. Ashkinazi, S.G. Ryzhkov, A.V. Vlasov, A.V. Khomich. Diam. Relat. Mater., 62, 49 (2016).

[19] A.L. Vikharev, M.A. Lobaev, A.M. Gorbachev, D.B. Radishev, V.A. Isaev, S.A. Bogdanov. Mater. Today Comm., 22, 100816 (2020).

[20] Z. Minglong, X.Yiben, W. Linjun, S. Hujiang, G. Beibei. Solid State Commun., 130, 551 (2004).

[21] F. Schirru, D. Chokheli, M. Kis. Diam. Relat. Mater., 49, 96 (2014).

[22] A. Balducci, M. Marinelli, E. Milani, M.E. Morgada, G. Pucella, M. Scoccia, A. Tucciarone, G. Verona-Rinati, M. Angelone, M. Pillon, R. Potenza, C. Tuve. Diam. Relat. Mater., 15, 292 (2006).

[23] M. Pomorski, C. Delfaure, N. Vaissiere, H. Bensalah, J. Barjon, M.A. Pinault-Thaury, D. Tromson, P. Bergonzo. Phys. Stat. Sol. A, 212 (11), 2553 (2015). DOI: $10.1002 /$ pssa.201532230

[24] T. Shimaoka, J.H. Kankeko, M. Tsubota, H. Shimmyo, H. Watanabe, A. Chayahara, H. Umezawa, S. Shikata. Europhys. Lett., 113 (6), 62001 (2016).

[25] S.V. Chernykh, S.A. Tarelkin, A.V. Chernykh, S.Yu. Troschiev, N.V. Luparev, N.V. Kornilov, D.V. Teteruk, S.A. Terentiev, V.D. Blank, A.V. Antipov, A.P. Chubenko, Yu.N. Glybin, N.I. Polushin, S.I. Didenko. Instrum. Exp. Tech. 62 (4), 473 (2019).

[26] Н.Б. Родионов, А.Ф. Паль, А.П. Большаков, В.Г. Ральченко, Р.А. Хмельницкий, В.А. Дравин, С.А. Малыхин, И.В. Алтухов, М.С. Каган, С.К. Папроцкий. Радиотех. и электрон., 63 (7), 750 (2018).

[27] S. Almaviva, M. Marinelli, E. Milani, G. Prestopino, A. Tucciarone, C. Verona, G. Verona-Rinati, M. Angelone, M. Pillon. Nucl. Instrum. Meth. Phys. Res., A 612, 580 (2010).

[28] Н.Б. Родионов, В.Н. Амосов, К.К. Артемьев, С.А. Мещанинов, В.П. Родионова, Р.А. Хмельницкий, В.А. Дравин, А.П. Большаков, В.Г. Ральченко. Атомная энергия, 121 (2), 98 (2016).

[29] Н.Б. Родионов, В.Н. Амосов, С.А. Мещанинов, А.Ф. Паль, В.П. Родионова, А.Г. Трапезников. Приборы и техника эксперимента, 5, 77 (2016).

[30] A.P. Bolshakov, V.G. Ralchenko, G. Shu, B. Dai, V.Yu. Yurov, E.V. Bushuev, A.A. Khomich, A.S. Altakhov, E.E. Ashkinazi, I.A. Antonova, A.V. Vlasov, Y.Y. Sizov, S.K. Vartapetov, V.I. Konov, J. Zhu. Mater. Today Commun., 25, 101635 (2020). DOI: 10.1016/j.mtcomm.2020.101635
[31] R. Samlenski, C. Haug, R. Brenn, C. Wild, R. Locher, P. Koidl. Appl. Phys. Lett., 67, 2798 (1995).

[32] M.N.R. Ashfold, J.P. Goss, B.L. Green, P.W. May, M.E. Newton, C.V. Peaker. Nitrogen Diamond. Chem. Rev., 120 (12), 5745 (2020).

[33] A. Tallaire, M. Kasu, K. Ueda, T. Makimoto. Diam. Relat. Mater., 17 (1), 60 (2008).

[34] A.T. Collins. J. Phys.: Condens. Matter., 14, 3743 (2002).

[35] J.P. Goss, P.R. Briddon, R. Jones, S. Sque. Diam. Relat. Mater., 13, 684 (2004).

[36] N. Aslam, G. Waldherr, P. Neumann, F. Jelezko, J. Wrachtrup. New J. Phys., 15, 013064 (2013).

[37] P.-N. Volpe, P. Muret, F. Omnes, J. Achard, F. Silva, O. Brinza, A. Gicquel. Diam. Relat. Mater., 18, 1205 (2009).

[38] H. Luo, K.M. Ajmal, W. Liu, K. Yamamura, H. Deng. Int. J. Extrem. Manuf., 3, 022003 (2021).

[39] H.B. Dyer, F.A. Raal, L. DuPreez, J.H.N. Loubser. Philos. Mag., 11, 763 (1965).

[40] R. Jones, J.P. Goss, P.R. Briddon. Phys. Rev. B: Condens. Matter. Mater. Phys., 80, 033205 (2009). 